\title{
Limit theorems for semi-Markov processes
}

\section{K.B. Athreya and P.E. Ney}

\begin{abstract}
A new construction of regeneration times is exploited to prove ergodic and renewal theorems for semi-Markov processes on general state spaces. This work extends results of the authors in Ann. Probability (6 (1978), 788-797).
\end{abstract}

\section{Introduction}

This note is a continuation of [2] and [3], where we introduced a construction of regeneration times to show that recurrent Markov chains on general state spaces act as if they had a single recurrence point which is visited infinitely often. This device was used to give renewal theoretic proofs of ergodic and renewal theorems for Markov and semi-Markov chains. The semi-Markov results were proved under a strong aperiodicity condition on the underlying Markov chain, and a further strong restriction on the distributions of sojourn times in a state. Our objective here is to remove some of these restrictions.

Consider a space $S$ with a o-algebra of subsets $S$. Let $\left\{x_{n} ; n=0,1, \ldots\right\}$ be a Markov chain on $(S, S)$ with homogeneous transition function $P(s, E), s \in S, E \in S$, and let $\left\{G_{x y}(\cdot) ; x, y \in S\right\}$ be a family of distribution functions on $R^{+}=[0, \infty)$. Given a realization $\left\{x_{n}=x_{n} ; n=0,1, \ldots\right\}$ of the chain, generate independent random variables $\left\{L_{n} ; n=0,1, \ldots\right\}$ such that

Received 21 August 1978. This work was done while the authors were visiting the University of Western Australia. They would like to thank Dr Richard L. Tweedie and the Department of Mathematics of the University of Western Australian for their hospitality. 


$$
\text { (1.1) } P\left\{L_{i} \leq t \mid X_{n}=x_{n}, n=0,1, \ldots\right\}=G_{x_{i}, x_{i+1}}(t), t \geq 0 \text {. }
$$

Set

$$
W(t)=(z(t), A(t))=\left\{\begin{array}{ccc}
\left(x_{0}, t\right) & \text { when } & 0 \leq t<L_{0} \\
\left(x_{1}, t-L_{0}\right) & \text { when } & L_{0} \leq t<L_{0}+L_{1} \\
\vdots & \vdots & \vdots \\
\left(x_{k}, t-\sum_{0}^{k-1} L_{i}\right) & \text { when } & \sum_{0}^{k-1} L_{i} \leq t<\sum_{0}^{k} L_{i} \\
\vdots & &
\end{array}\right.
$$

The process $\{W(t) ; 0 \leq t\}$ is a Markov process. $\{Z(t) ; 0 \leq t\}$ is called a semi-Markov process, and $\{A(t) ; 0 \leq t\}$ is its associated age process. Also $\left\{X_{n}\right\}$ is referred to as the state process, $\left\{L_{n}\right\}$ as its "sojourn" times, and $\left\{X_{n}, L_{n-1}\right\}$ as a semi-Markov chain. (Note that this pair chain is actually a Markov chain; the joint distribution of $\left(x_{n+1}, L_{n}\right)$ depends only on $x_{n}$. ) The objective is to determine the limiting behaviour of $W(t)$; more specifically of functions of the form $E f(W(t))$ and $E\left(\sum_{n=0}^{\infty} g\left(X_{n}, t-\sum_{i=0}^{n} L_{i}\right)\right)$, for reasonable classes of functions $f$ and $g$.

The key hypothesis is designed to guarantee the required recurrence structure.

HYPOTHESIS $H_{k}$. (i) There exists a set $A \in S$ such that for some integer $k$,

$$
p_{x}\left\{X_{n k} \in A \text { for some } n \geq 1\right\}=1, x \in S \text {. }
$$

(ii) There exists a probability measure $\varphi$ on $(S \cap A, S \cap A), a$ family of probability measures $\mu(x, \cdot)$ on $B\left(R^{+}\right)$for $x \in A$, and $a$ number $\lambda \in(0,1)$, such that

$$
P_{x}\left(X_{k} \in E, \sum_{i=0}^{k-1} L_{i} \in D\right) \geq \lambda \varphi(E \cap A) \mu(x, D)
$$

for all $x \in A, D \in B\left(R^{+}\right)$. 
We then have the following

THEOREM. Assume $\mathrm{H}_{\mathrm{k}}$. Then

(a) there exists a o-finite, invariant measure $v$ for $P$ which is unique up to multiplicative constants;

(b) if furthermore

(i) $m \equiv \int m(x) v(d x)<\infty$, where $m(x)=E_{x_{0} L_{0}}$, (ii) $P\left(\sum_{0}^{k-1} L_{i} \leq u\right)=F_{k}(u)$ is non-lattice, and (iii) $f: S \times R^{+} \rightarrow R^{+}$is bounded, measurabie, and $v\{x: f(x, t)$ is discontinuous for some $t\}=0$,

then

(1.4) $\lim E f(W(t))$

$t \rightarrow \infty$

$\left.\left.=\iint_{S}\left(\int_{0}^{\infty} f(x, u) P_{x}\left(L_{0}>u\right) d u\right) v(d x)\right) / \iint_{S}\left(\int_{0}^{\infty} P_{x}\left(L_{0}>u\right) d u\right) v(d x)\right) ;$

(c) if (b) (i) and (ii) hold, and $g(x, t): S \times R^{+}+R$ is bounded, measurable, continuous in $t$, and satisfies

$$
g(x, t) \rightarrow 0 \text { almost surely (v) as } t \rightarrow \infty,
$$

$$
\int_{S} \sum_{n=0}^{\infty} \sup _{n h \leq t<(n+1) h} g(x, t) v(d x)<\infty,
$$

and

$$
\int\left\{\sup _{t}|g(x, t)|\right\} v(d x)<\infty,
$$

then as $t \rightarrow \infty$,

$$
E_{\varphi} \sum_{n=0}^{\infty} g\left(X_{n}, t-\sum_{i=0}^{n-1} L_{i}\right) \rightarrow \frac{1}{m} \int_{0}^{\infty} \int_{S} g(x, t) v(d x) d t .
$$

REMARKS. 1. The theorem extends results of [2] in two ways, First, the distributions $G_{x y}(\cdot)$ were allowed to depend only on $x$ (not $y$ ) in the earlier work; that is, there the sojourn times depend only on the 
state the chain is "coming from". Secondly, only the case $k=1$ is admitted in [2].

2. The awkward conditions (1.5)-(1.7) are designed to assure the direct Riemann integrability of certain functions related to $g$. These conditions can be eliminated at the expense of strengthening the smoothness hypothesis on $F_{k}$ in (b) (ii). (see the related discussion in Arjas, Nummel in, and Tweedie [1], and Athreya and Ney [4].)

3. Nummel in [7], [8], has proved many results related to this subject, including one like the above theorem under apparently slightly stronger hypotheses. He also uses regeneration methods, but treats the semi-Markov process as a Markov chain on the enlarged state space $S \times R$, and applies discrete renewal theory to this chain. We work instead with the continuous time process $(z(t), A(t))$ and use renewal theory on $R$ to draw our conclusions. This approach seems to us to yield somewhat more transparent proofs.

4. Nummelin has also shown (see [7]) that a condition very close to $\mathrm{H}_{\mathrm{k}}$ is always satisfied (for some $k$ ), provided the semi-Markov chain satisfies a weak irreducibility condition.

5. That (1.8) is in fact a "renewal theorem", can be seen by taking $g(x, t)$ of the form $\chi_{A}(x) \chi_{I}(t)$ for $A \in S$, and $I \subset R^{+}$an interval. As usual in such results, if $F_{k}(\cdot)$ is lattice, then there is a lattice version of (1.8).

6. Similarly (1.4) can be seen to imply the convergence of $P\{2(t) \in E\}, E \in S$.

7. Since (a) asserts the uniqueness of the invariant measure, one can use any measure $\pi(\cdot)$ that is invariant with respect to $P$ in place of $v$.

8. If $k=1$ and $G_{x y}$ depends only on $x$, then $H_{1}$ automatically holds.

9. The result (c) of the theorem carries over without difficulty to the "two sided" case when the $G_{x y}(\cdot)$ are distributions on $(-\infty, \infty)$, along the lines of Theorem 4.1 of [2]. 
10. Renewal theorems of the type in (c) of the theorem under different hypotheses have been proved by Jacod [5] and Kesten [6].

\section{Proof of the theorem}

Hypothesis $\mathrm{H}_{\mathrm{k}}$ (ii) applied to $D=R^{+}$implies that the Markov chain $\left\{x_{n k} ; n=0,1, \ldots\right\}$ is $(A, \lambda, \varphi, 1)$-recurrent in the sense of Definition (2.2) of [3]. Hence, by Theorem (6.1) of that paper, there exists a unique (up to multiplicative constants) invariant measure, say $v_{k}$, for $P$. It is now easy to verify that

$$
v=v_{k}+v_{k} P+\ldots+v_{k} P^{k-1}
$$

is a (necessarily unique) invariant measure for $P$.

As in the earlier work [2], [3], the basic idea behind the proof is an appropriate

REGENERATION LEMMA. If $\mathrm{H}_{\mathrm{k}}$ holds for some $k \geq 1$, then there exists a rondom time $N$ such that $P_{x}(N<\infty)=1$ for alz $x \in S$, and

$$
\begin{aligned}
P_{x}\left\{X_{N k} \in E, \sum_{(N-1) k}^{N k-1} L_{i} \in D \mid X_{j k}, \sum_{i=0}^{j k} L_{i}, j\right. & =0,1, \ldots, N-1\} \\
& =\varphi(E \cap A) \mu\left(X_{(N-1) k}, D\right),
\end{aligned}
$$

almost surely.

Proof. Let $U_{j}=L_{j k}+\ldots+L_{(j+1) k-1}$, and consider the "skeleton" semi-Markov chain $\left\{\left(x_{j k}, U_{j-1}\right), j=1,2, \ldots\right\}$ ( $k$ is fixed throughout $)$. Whenever $X_{j k} \in A$ for some $j$ (say $X_{j k}=x \in A$ ), randomize the next transition of the chain as follows:

(i) with probability $p \quad(0<p<\lambda=$ the constant in (1.3)) distribute $\left(X_{(j+1) k}, U_{j}\right)$ over $A \times R^{+}$independently with distributions $\varphi(\cdot)$ and $\mu(x, \cdot)$ respectively;

(ii) with probability $(1-p)$ distribute $\left(x_{(j+1) k}, U_{j}\right)$ over the entire state space $S \times R^{+}$according to a transition function $Q(x, \cdot)$, chosen so that the overall transition probabilitities of the chain 
$\left\{\left(x_{j k}, U_{j-1}\right)\right\}$ remain unchanged. This is accomplished by defining $Q$ so that

$$
P^{k}(x, E \times D)=p \varphi(E \cap B) \mu(x, D)+(1-p) Q(x, E \times D) .
$$

That this is in fact possible, follows from (1.3). Now since $A$ is visited by $\left\{x_{n k}\right\}$ infinitely often, and each time, with probability $p>0$, the next transition is distributed independently according to $(\varphi, \mu)$, this event will ultimately occur at some time $N<\infty$ almost surely. This proves the lemma. (The reader wishing to see a more detailed argument is referred to the proof of (3.1) in [3], which contains a careful proof of a special case of the above lemma.)

COROLLARY 1. There exists a sequence of rondom times $N_{1}, N_{2}, \ldots$ for which (2.1) holds.

COROLLARY 2. Let $T=\sum_{0}^{N k-1} L_{i}$ and $Z=X_{N k} \cdot$ Then $Z$ and $T$ are independent random variables.

Proof. Let $f$ and $g$ be bounded, measurable functions on $S$ and $R^{+}$, respectively. Then

$$
\begin{aligned}
E f(Z) g(T) & =E\left\{E\left[f(Z) g(T) \mid\left(x_{j k}, U_{j-1}\right), j=0, \ldots, N-1\right]\right\} \\
& =E\left\{E\left[f\left(X_{N k}\right) g\left(\sum_{0}^{N-2} U_{i}+U_{N-1}\right) \mid\left(X_{j k}, U_{j-1}\right), j=0, \ldots, N-1\right]\right\} \\
& =E\left\{\int_{A} f(y)_{\varphi}(d y) \int_{R} g\left(\sum_{0}^{N-2} U_{i}+u\right) \mu(X(N-1) k, d u)\right\} .
\end{aligned}
$$

Taking the first integral outside the expectation, this equals $E f(Z) E g(T)$, proving the corollary. Before turning to the main part of the proof of the theorem, we prepare one more

PROPOSITION. Let $h: S \times S \rightarrow R$ be bounded and measurable, and $\tilde{h}(x)=E_{x} h\left(X_{0}, X_{1}\right)$. Then

$$
E_{\varphi} \sum_{n=0}^{N k-1} h\left(x_{n}, x_{n+1}\right)=\int_{S} \tilde{h}(x) \cup(d x)
$$

where $\nu(\cdot)$ is the stationary measure whose existence was asserted in part 
(a) of the theorem.

Proof.

$$
\begin{aligned}
E_{\varphi} \sum_{n=0}^{N k-1} h\left(x_{n}, x_{n+1}\right) & =E_{\varphi} \sum_{j=0}^{N-1} \sum_{n=j k}^{(j+1) k-1} h\left(x_{n}, x_{n+1}\right) \\
& =E_{\varphi} \sum_{j=0}^{\infty} \sum_{n=j k}^{(j+1) k-1} h\left(x_{n}, x_{n+1}\right) x_{(N>j)} .
\end{aligned}
$$

Now extend the chain $\left\{x_{n}\right\}$ to a chain $\left\{x_{n}, \delta_{n}\right\}, n \geq 0$, where $\left\{\delta_{n} ; n \geq 0\right\}$ is a sequence of independent "coin tossing" variables with $P($ Head $)=P\left(\delta_{n}=1\right)=1-P\left(\delta_{0}=0\right)=p$. The evolution of $\left\{x_{n}\right\}$ is independent of $\left\{\delta_{n}\right\}$ except when $X_{n k} \in A$, at which time the randomization described in the proof of the regeneration lemma takes place. Thus the random variable $X_{(N>j)}$ is completely determined by the history $\left\{\left(x_{i}, \delta_{i}\right), i=1, \ldots, j k\right\}$, and hence conditioned on this history (say $\left.F_{j k}\right)\left(x_{j k}, \ldots, x_{(j+1) k}\right)$, and $x_{(N>j)}$ are trivially independent. Thus for any bounded, measurable $\psi: S^{k} \rightarrow R$, we have

$$
\begin{aligned}
E \psi\left(x_{j k}, \ldots, x_{(j+1) k}\right) x_{(N>j)} & =E\left\{E\left[\psi x \mid F_{j k}\right]\right\}=E\left\{x E\left[\psi \mid F_{j k}\right]\right\} \\
& =E\left\{x_{N>j} E\left[\psi\left(x_{j k}, \ldots, x_{(j+1) k}\right\} \mid x_{j k}\right]\right\} \\
& \equiv E\left\{\tilde{\psi}\left(x_{j k}\right) x_{(N>j)}\right\},
\end{aligned}
$$

where we have let

$$
\tilde{\psi}(x)=E\left\{\psi\left(x_{0}, \ldots, x_{k}\right\} \mid x_{0}=x\right\}
$$

Also let

$$
\begin{aligned}
H(x)=E_{x} \sum_{n=0}^{k-1} h\left(x_{n}, x_{n+1}\right)= & \sum_{n=0}^{k-1} E_{x} \tilde{h}\left(x_{n}\right) \\
& \left(E_{x} h\left(x_{n}, x_{n+1}\right)=E_{x} E_{X_{n}} h\left(x_{n}, x_{n+1}\right)=E_{x}\left(\tilde{h}\left(x_{n}\right)\right)\right) .
\end{aligned}
$$

Then 


$$
\begin{aligned}
E_{\varphi} \sum_{n=0}^{N k-1} h\left(x_{n}, x_{n+1}\right) & =E_{\varphi} \sum_{j=0}^{N-1} \sum_{n=j k}^{(j+1) k-1} h\left(x_{n}, x_{n+1}\right) \\
& =E_{\varphi} \sum_{j=0}^{N-1} H\left(x_{j k}\right)=E_{\varphi} \sum_{j=0}^{\infty} H\left(x_{j k}\right) x_{N>j} .
\end{aligned}
$$

By Theorem (6.1) of [3] we know that an invariant measure for $P^{\mathcal{K}}$ is given by $v_{k}(E)=E_{\varphi} \sum_{j=0}^{N-1} \chi_{E}\left(X_{j k}\right)$, and hence (2.4) equals

$$
\begin{aligned}
\int_{S} H(x) \nu_{k}(d x) & =\int_{S} \tilde{h}(y) \sum_{n=0}^{k-1} \int_{S} P^{n}(x, d y) \nu_{k}(d y) \\
& =\int_{S} \tilde{h}(y) \nu(d y) \text { by }(2.1) .
\end{aligned}
$$

This, with (2.4), is (2.3), proving the proposition.

We now turn to the main part of the proof of part $(b)$ of the theorem. Since $P_{x}(T<\infty)=1$ for all $x \in S$, it is sufficient to prove this result for the case when the initial state $X_{0}$ is distributed according to $\varphi$. Let $m(t)=E f(W(t))$, and $a(t)=E\{f(W(t)\} ; T>t\}$, where $f$ satisfies the conditions of part $(b)$ of the theorem, and $T$ is as in Corollary 2. Due to the independence assertion of that corollary, $T$ is a "regeneration time", at which the chain $\left\{X_{n}\right\}$ undergoes a transition and is distributed over $A$ according to $\varphi$, independent of the history of the process up to that time. Hence $m(\cdot)$ satisfies the one-dimensional renewal equation

$$
m(t)=a(t)+\int_{0}^{t} m(t-u) d F(u)
$$

where

$$
F(u)=P_{\varphi}(T \leq u)
$$

The direct Riemann integrability of $a(\cdot)$ under the hypotheses on $f(\cdot)$ now follows exactly as in the proof of Theorem (3.1) of [2]. Hence, since $F(\cdot)$ is non-lattice (note that this is a little weaker than the hypothesis $F_{k}$ non-lattice), we have by the renewal theorem that 


$$
m(t) \rightarrow\left(E_{\varphi} T\right)^{-1} \int_{0}^{\infty} a(y) d y \text {, as } t \rightarrow \infty \text {. }
$$

It thus remains only to identify the limit. To this end we write

$$
\begin{aligned}
\int_{0}^{\infty} a(t) d t & =E_{\varphi} \int_{0}^{T} f(W(u)) d u \\
& =E_{\varphi} \sum_{n=0}^{N k-1} \int_{0}^{L} f\left(x_{n}, u\right) d u \\
& =E_{\varphi}\left\{E_{\varphi}\left[\sum_{n=0}^{N k-1} \int_{0}^{L} f\left(x_{n}, u\right) d u \mid x_{0}, \ldots, x_{N-1}, N\right]\right\} \\
& =E_{\varphi} \sum_{n=0}^{N k-1} h\left(x_{n}, x_{n-1}\right),
\end{aligned}
$$

where

$$
\begin{aligned}
h(x, y) & =E\left[\int_{0}^{L} f\left(x_{0}, u\right) d u \mid x_{0}=x, x_{1}=y\right] \\
& =\int_{0}^{\infty} f(x, u)\left[1-G_{x y}(u)\right] d u .
\end{aligned}
$$

Applying (2.3) of the proposition, we see that

$$
\int_{0}^{\infty} a(t) d t=\int_{S} \tilde{h}(x) \nu(d x),
$$

where

$$
\begin{aligned}
\tilde{h}(x) & =E_{x} E h\left(X_{0}, X_{1}\right) \\
& =\int_{S} \int_{0}^{\infty} f(x, u)\left[1-G_{x y}(u)\right] d u P(x, d y) \\
& =\int_{0}^{\infty} f(x, u) P_{x}\left(L_{0}>u\right) d u .
\end{aligned}
$$

Thus $\int a(t) d t$ equals the numerator in (1.4). For the denominator, we observe from (2.6) that $E_{\varphi} T=\int_{0}^{\infty} a_{1}(t) d t$, where $a_{1}(\cdot)$ is $a(\cdot)$ for the 
special case when $f(x, t) \equiv 1$. This implies part $(b)$ of the theorem.

Finally, turning to part (c), we again observe that we need only consider the case when $X_{0}$ has distribution $\varphi$. Let $K(t) \equiv E_{\varphi} \sum_{n=0}^{N k-1} g\left(x_{n}, t-\sum_{i=0}^{n-1} L_{i}\right)$ and $T_{j}=\sum_{N_{j} k}^{N+1} L_{i}$, where $\left\{N_{j} ; j \geq 0\right\}$ are the successive regeneration times for $\left\{X_{n k}\right\}$. Then $\left\{T_{j} ; j \geq 0\right\}$ are independent identically distributed as $T$ in Corollary 2 , and are regeneration times, in the previously described sense, for the continuous time process. Hence

$$
\begin{aligned}
E_{\varphi} \sum_{n=N}^{N+1} \sum_{j}^{k-1} g\left(X_{n}, t-\sum_{i=0}^{n-1} L_{i}\right) & =E_{\varphi} \sum g\left(X_{n}, t-\sum_{i=0}^{j-1} T_{i}-\sum_{i=N}^{n-1} L_{j}\right) \\
& =E_{\varphi} K\left(t-\sum_{i=0}^{j-1} T_{i}\right) .
\end{aligned}
$$

Thus

$$
\begin{aligned}
M(t) \equiv E_{\varphi} \sum_{n=0}^{\infty} g\left(X_{n}, t-\sum_{i=0}^{n-1} L_{i}\right) & =E_{\varphi} \sum_{j=0}^{\infty} \sum_{n=N}^{N+1} \sum_{j}^{k-1} g\left(X_{n}, t-\sum_{i=0}^{n} L_{i}\right) \\
& =E_{\varphi} \sum_{j=0}^{\infty} g\left(X_{n}, t-\sum_{i=0}^{j-1} T_{i}\right) .
\end{aligned}
$$

But now we are ready to apply the one dimensional renewal theorem (exactly as in Section 4 of [2]), to conclude that if $K(\cdot)$ is direct Riemann integrable, then

$$
M(t) \rightarrow\left(E_{\varphi} T\right)^{-1} \int_{0}^{\infty} k(u) d u \text { as } t \rightarrow \infty
$$

The hypotheses on $g$ are exactly as those in [2], and are designed to assure this direct Riemann integrability.

To identify the limit in (1.8) we write 


$$
\begin{aligned}
\int_{0}^{\infty} K(t) d t & =E_{\varphi} \sum_{n=0}^{N k-1} \int_{0}^{\infty} g\left(X_{k}, t-\sum_{i=0}^{n-1} L_{i}\right) d t \\
& =E_{\varphi} \sum_{n=0}^{N k-1} \int_{0}^{\infty} g\left(X_{k}, t\right) d t,
\end{aligned}
$$

(we may define $g(x, t)=0$ for $t<0$ )

$$
=\int_{S} \int_{0}^{\infty} g(x, t) v(d x) d t .
$$

We have already seen that $\left.E_{\varphi}(T)=m(b)(i)\right)$. This completes the proof of the theorem.

We again ask the reader to observe that the above proof of (c) in no way depends on the non-negativity of the $L_{i}$ 's.

\section{References}

[1] Elja Arjas, Esa Numelin, Richard L. Tweedie, "Uniform limit theorems for non-singular renewal and Markov renewal processes", J. Appl. Probability 15 (1978), 112-125.

[2] K.B. Athreya, D. MCDonald and P.E. Ney, "Limit theorems for semiMarkov processes and renewal theory for Markov chains", Arn. Probability 6 (1978), 788-797.

[3] K.B. Athreya and P.E. Ney, "A new approach to the limit theory of recurrent Markov chains", Trans. Amer. Math. Soc. (to appear).

[4] K.B. Athreya and P.E. Ney, "Regeneration methods for Markov chains and related processes", under preparation.

[5] J. Jacod, "Théorème de renouvellement et classification pour les chaînes semi-markoviennes", Ann. Inst. H. Poincaré Sect. B (N.S.) 7 (1971), 83-129.

[6] Harry Kesten, "Renewal theory for functionals of a Markov chain with general state space", Arm. Probability 2 (1974), 355-386. 
[7] Esa Nummelin, "A splitting technique for o-recurrent Markov chains" (Report-HTKK-MAT-A80, Institute of Mathematics, Helsinki University of Technology, Espoo, Finland, 1976).

[8] Esa Nummelin, "Uniform and ratio limit theorems for Markov renewal and semi-regenerative processes on a general state space" (Report HTKK-MAT-A98, Institute of Mathematics, Helsinki University of Technology, Espoo, Finland, 1977).

Indian Institute of Science,

Bangalore,

India;

Department of Mathematics,

University of Wisconsin,

Madison,

Wisconsin,

USA. 